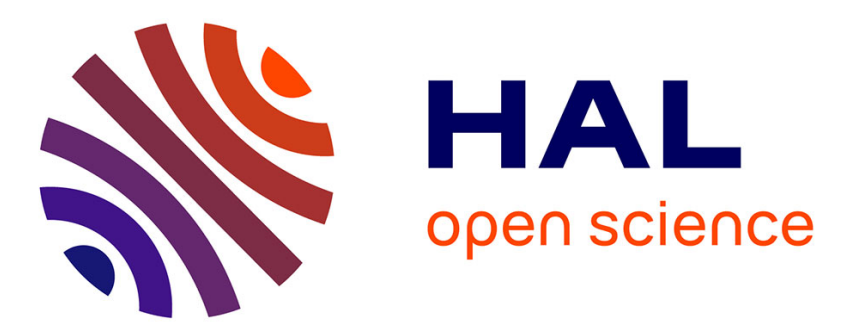

\title{
On the degree evolution of a fixed vertex in some growing networks
}

Mathias Lindholm, Thomas Vallier

\section{To cite this version:}

Mathias Lindholm, Thomas Vallier. On the degree evolution of a fixed vertex in some growing networks. Statistics and Probability Letters, 2011, 81 (6), pp.673. 10.1016/j.spl.2011.02.015 . hal00734953

\section{HAL Id: hal-00734953 \\ https://hal.science/hal-00734953}

Submitted on 25 Sep 2012

HAL is a multi-disciplinary open access archive for the deposit and dissemination of scientific research documents, whether they are published or not. The documents may come from teaching and research institutions in France or abroad, or from public or private research centers.
L'archive ouverte pluridisciplinaire HAL, est destinée au dépôt et à la diffusion de documents scientifiques de niveau recherche, publiés ou non, émanant des établissements d'enseignement et de recherche français ou étrangers, des laboratoires publics ou privés. 


\section{Accepted Manuscript}

On the degree evolution of a fixed vertex in some growing networks

Mathias Lindholm, Thomas Vallier

PII:

$$
\text { S0167-7152(11)00061-7 }
$$

DOI:

Reference: $\quad$ STAPRO 5913

To appear in: $\quad$ Statistics and Probability Letters

Received date: 26 November 2009

Revised date: 15 February 2011

Accepted date: 15 February 2011

Please cite this article as: Lindholm, M., Vallier, T., On the degree evolution of a fixed vertex in some growing networks. Statistics and Probability Letters (2011),

doi:10.1016/j.spl.2011.02.015

This is a PDF file of an unedited manuscript that has been accepted for publication. As a service to our customers we are providing this early version of the manuscript. The manuscript will undergo copyediting, typesetting, and review of the resulting proof before it is published in its final form. Please note that during the production process errors may be discovered which could affect the content, and all legal disclaimers that apply to the journal pertain. 


\title{
On the degree evolution of a fixed vertex in some growing networks
}

\author{
Mathias Lindholm* Thomas Vallier ${ }^{\dagger}$
}

February 18, 2011

\begin{abstract}
Two preferential attachment type graph models which allow for dynamic addition/deletion of edges/vertices are considered. The focus of this paper is on the limiting expected degree of a fixed vertex. For both models a phase transition is seen to occur, i.e. if the probability with which edges are deleted is below a model specific threshold value, the limiting expected degree is infinite, but if the probability is higher than the threshold value, the limiting expected degree is finite. In the regime above the critical threshold probability, however, the behaviour of the two models may differ. For one of the models a non-zero (as well as zero) limiting expected degree can be obtained whilst the other only has a zero limit. Furthermore, this phase transition is seen to occur for the same critical threshold probability of removing edges as the one which determines whether the degree sequence is of power-law type or if the tails decays exponentially fast.
\end{abstract}

\section{Introduction}

During the last decade there has been much interest in the study of large-scale networks. Real world networks such as the Internet and citation networks have been shown to exhibit power law degree sequences meaning that the proportion of vertices with degree $k$ decays as $k^{-\gamma}$ for some $\gamma>0$. The most famous and maybe the most studied model of real world networks is the preferential attachment model proposed by Barabási and Albert (Barabási and Albert, 1999) which later was defined and analysed rigourously by Bollobás et al. (2001). In this model, at each time step, a new vertex is introduced together with an edge attaching the new vertex to a previous one with a probability proportional to the degree. This mechanism can be shown to generate a power-law degree sequence with exponent $\gamma=3$, see Bollobás et al. (2001). For robustness of the model under deletion of vertices/edges, see Bollobás and Riordan (2003).

In the present paper we analyse two generalisations of the preferential attachment model. The first model is introduced in Deijfen and Lindholm (2009), a model where edges can be added/deleted dynamically over time and the second is introduced in Cooper et al. (2004) where vertices as well as edges can be added/deleted dynamically. For these models we derive results concerning the evolution of the expected degree of a single fixed vertex, and in particular, both models reveal a phase transition which is dependent on the probability of removing edges. That is, if the probability of removing edges is below a model specific threshold the limiting expected degree is infinite, and if the probability is above the critical threshold the limiting expected degree is finite (always zero for the model introduced in Cooper et al. (2004)). For the model of Deijfen and Lindholm (2009), this phase transition occurs at the same critical edge probability as for the phase transition for the degree sequence, i.e. where the degree sequence

\footnotetext{
*Department of Mathematics, Mathematical statistics, Uppsala University, Uppsala, Sweden

${ }^{\dagger}$ Centre for Mathematical Sciences, Mathematical statistics, Lund University, Lund, Sweden
} 
changes from power-law to exponential decay. Under a certain parametrisation a sub-model of the model of Cooper et al. (2004) coincides with a certain sub-model of the one treated in Deijfen and Lindholm (2009) which hence, partially, establishes the same type of phase transition of the degree sequence in the model of Cooper et al. (2004). Moreover, for the general model of Cooper et al. (2004), under the restriction of not deleting vertices, the critical edge probability of removing edges which determines the phase transition of the expected limiting degree of a fixed vertex is obtained. Eventhough the phase transition of the degree sequence is not treated in Cooper et al. (2004) the results obtained there indicate that such a phase transition may occur and that the behaviour of the degree sequence is determined by the same critical edge probability which determines the phase transition of the expected limiting degree of a fixed vertex.

\section{The model of Deijfen and Lindholm}

We consider a graph process $(G(t))_{t \geq 1}$ consisting of graphs $(V(t), E(t))$. Let $v_{t}=|V(t)|$ and $e_{t}=$ $|E(t)|$. We will throughout denote the degree of vertex $u$, born at $s \leq t$, at time $t$ by $d_{t}^{s}(u)$. Occasionally we will make use of the notation $d_{t}^{\bullet}(u)$ when we only need to know that a particular vertex $u$ is born before $t$.

To initialise the process, we start with $G(1)$ consisting of an isolated vertex with a loop attached to it. The graph is constructed recursively and at time $t+1$ the possible steps are the following:

1. With probability $\pi_{1}>0$ a vertex $u$ is introduced with an edge attached to it. The edge is connected to an existing vertex $w$ with probability proportional to its degree.

2. With probability $\pi_{2}$ an edge is added between a vertex chosen proportionally to its degree and another vertex chosen uniformly at random.

3. With probability $\pi_{3}=1-\pi_{1}-\pi_{2}$ an edge chosen uniformly is deleted.

If $e_{t}=0$ two things can occur, either a new vertex with a loop is introduced with probability $\pi_{1}$ or with probability $1-\pi_{1}$ an edge attached to two uniformly chosen vertices is added.

In the following the expected degree of a fixed vertex is studied and it is shown that a phase transition occurs at $\pi_{3}^{c r}=\frac{1}{3}$ where the expected degree changes from being infinite to being finite. As mentioned above, it is shown in Deijfen and Lindholm (2009) that the phase transition in the degree sequence occurs at the same critical edge probability.

Before we proceed with the computation of the expected degree of a single vertex some auxiliary results are needed.

\subsection{Number of vertices in the graph at time $t$}

Consider the following random variables:

$$
I_{i}=\left\{\begin{array}{l}
1, \quad \text { with probability } \pi_{1}, \\
0, \quad \text { with probability } 1-\pi_{1} .
\end{array}\right.
$$

Hence $I_{i}=1$ corresponds to the addition of a vertex and $I_{i} \sim \operatorname{Be}\left(\pi_{1}\right)$. Thus, $N(t)=\sum_{i=1}^{t} I_{i} \sim \operatorname{Bin}\left(t, \pi_{1}\right)$ since all $I_{i}$ are i.i.d. It is also worth noting that $v_{t}={ }_{d} N(t)$. Due to the construction of $N(t)$ it also holds that $\frac{1}{t} N(t)$ converges a.s. (almost surely) to $\pi_{1}$ as $t \rightarrow \infty$ according to the strong law of large numbers. Moreover, by using Markov's inequality together with $\operatorname{Var}(N(t))=\pi_{1}\left(1-\pi_{1}\right) t$ we get that

$$
\mathbb{P}\left\{\left|N(t)-\pi_{1} t\right|>t^{1 / 2+\epsilon}\right\} \rightarrow 0 \text { as } t \rightarrow \infty,
$$

that is, $v_{t}=N(t)=\pi_{1} t\left(1+\Theta\left(t^{-1 / 2+\epsilon}\right)\right)$ a.a.s. (asymptotically almost surely) for any $0<\epsilon<1 / 2$. 


\subsection{Number of edges in the graph at time $t$}

In order to obtain results on the number of edges in the graph at $t$ it is convenient to make use of that the edge process can be described as a simple random walk with reflecting barrier at 0 . Denote this process by $X_{0}(t)$ defined by the following transition probabilities

$$
X_{0}(t+1)= \begin{cases}X_{0}(t)+1, & \text { with probability } 1-\pi_{3} \\ X_{0}(t)-1, & \text { with probability } \pi_{3}\end{cases}
$$

if $X_{0}(t) \geq 1$ and

$$
X_{0}(t+1)=1
$$

if $X_{0}(t)=0$. If the rate of deletion, $\pi_{3}$, is greater or equal to $\frac{1}{2}$ it holds that

$$
\mathbb{P}\left\{e_{t}=0 \text { i.o. }\right\}=1 \text {. }
$$

The case when the rate of deletion, $\pi_{3}$, is smaller than $\frac{1}{2}$, corresponds a random walk with reflecting barrier where all the states are transient with a drift towards $+\infty$. But, due to transience we shall see that one can use a coupling argument to show that the random walk with reflecting barrier can be well approximated by a simple random walk, defined below, for large $t$. To start with, it follows that the return time to zero at time $2 s$ given that the process is started at zero is given by

$$
p_{0}^{2 s}:=\mathbb{P}\left(X_{0}(2 s)=0 \mid X_{0}(0)=0\right) \sim \frac{\left(4 \pi_{3}\left(1-\pi_{3}\right)\right)^{s}}{\sqrt{\pi s}},
$$

see e.g. Shiryaev $(1996)$ p. 588. Using this fact it follows that $\sum_{s \geq s_{0}} p_{0}^{2 s} \leq \frac{1}{\sqrt{\pi s_{0}}} \sum_{s \geq s_{0}}\left(4 \pi\left(1-\pi_{3}\right)\right)^{s} \leq$ $\frac{C}{\sqrt{s_{0}}}\left(4 \pi\left(1-\pi_{3}\right)\right)^{s_{0}} \leq\left(4 \pi\left(1-\pi_{3}\right)\right)^{s_{0}}$ for $s_{0}$ large. Thus the probability of hitting 0 after time $-\frac{2}{\log \left(4 \pi_{3}\left(1-\pi_{3}\right)\right)} \log n$ is

$$
\mathbb{P}\left\{\exists t \geq-\frac{2}{\log \left(4 \pi_{3}\left(1-\pi_{3}\right)\right)} \log n, e_{t}=0\right\}=O\left(\frac{1}{n^{2}}\right) .
$$

Thus, combining (2.4) together with the transience of $X_{0}$ implies that $X_{0}$ with a high probability will be in $\mathbb{Z}_{+}$for large $t$. Introducing the simple random walk $X(t)$ defined by the transition probabilities in (2.1) then $X_{0}(t)$ is bounded from below by $X(t)$. This fact together with (2.4) implies that

$$
\mathbb{P}\left\{\left|X_{0}(t)-X(t)\right| \geq-\frac{2}{\log \left(4 \pi_{3}\left(1-\pi_{3}\right)\right)} \log n\right\}=O\left(\frac{1}{n^{2}}\right) .
$$

Hence, for large $t$ the process $X_{0}$ will with a high probability be well approximated by $X$ in the sense defined in (2.5). Moreover, $X(t)=\sum_{i=1}^{t} J_{i}$ where all $J_{i}$ are i.i.d with

$$
J_{i}=\left\{\begin{aligned}
1, & \text { with probability } 1-\pi_{3}, \\
-1, & \text { with probability } \pi_{3} .
\end{aligned}\right.
$$

Thus $\mathbb{E}[X(t)]=\left(1-2 \pi_{3}\right) t$ which together with the strong law of large numbers yields that $\frac{X(t)}{t} \rightarrow$ $\left(1-2 \pi_{3}\right)$ a.s. as $t \rightarrow \infty$. Then, by using Markov's inequality together with that $\operatorname{Var} X(t)=4 \pi_{3}\left(1 \stackrel{t}{-} \pi_{3}\right) t$ it follows that

$$
\mathbb{P}\left\{\left|X(t)-\left(1-2 \pi_{3}\right) t\right|>t^{1 / 2+\epsilon}\right\} \rightarrow 0 \text { as } t \rightarrow \infty,
$$

that is, $X(t)=\left(1-2 \pi_{3}\right) t\left(1+\Theta\left(t^{-1 / 2+\epsilon}\right)\right)$ a.a.s. for any $0<\epsilon<1 / 2$. Notice that for large $t$ and large enough values of $n$ the concentration result of (2.5) can be made sharper than the bound in (2.6) and it hence still holds that

$$
2 e_{t}=2\left(1-2 \pi_{3}\right) t\left(1+\Theta\left(t^{-1 / 2+\epsilon}\right)\right)
$$

a.a.s. An important observation is that $2 e_{t}=\sum_{u \in V(t)} d_{t}^{\bullet}(u)$. Knowing this together with that $v_{t}=$ $\pi_{1} t\left(1+\Theta\left(t^{-1 / 2+\epsilon}\right)\right)$ holds a.a.s., we can focus on the expected degree of a vertex. 


\section{$2.3 \quad$ Expected degree of a vertex}

We first consider the case $\pi_{3}<\frac{1}{2}$. When analysing the evolution of the degree of a single vertex it is important to know when a particular vertex is added to the graph. For $s \neq t+1$ it holds that

$$
\begin{aligned}
\mathbb{E}\left[d_{t+1}^{s}(u)-d_{t}^{s}(u) \mid G(t)\right] & =\pi_{1} \frac{d_{t}^{s}(u)}{\sum_{w \in V(t)} d_{t}^{\bullet}(w)} \\
& +\pi_{2}\left(\frac{d_{t}^{s}(u)}{\sum_{w \in V(t)} d_{t}^{\bullet}(w)}+\frac{1}{v_{t}-1}\left(1-\frac{d_{t}^{s}(u)}{\sum_{w \in V(t)} d_{t}^{\bullet}(w)}\right)\right) \\
& -\pi_{3} \frac{d_{t}^{s}(u)}{e_{t}}
\end{aligned}
$$

and $\mathbb{E}\left[d_{s}^{s}(u)\right]:=1$. In (2.7) the expression following $\pi_{1}$ corresponds to the probability that a new edge is added to vertex $u$ and the expression following $\pi_{2}$ corresponds to the probability that a new edge is added to $u$ by either choosing $u$ as start or end vertex. The expression following $\pi_{3}$ corresponds to the probability that an edge is deleted from vertex $u$.

Using the concentration of the sum of the degrees and of the number of vertices combined with (2.7), we after some simplifications find that

$$
\begin{aligned}
\mathbb{E}\left[d_{t+1}^{s}(u)-d_{t}^{s}(u) \mid G(t)\right] & =\frac{1-3 \pi_{3}}{2\left(1-2 \pi_{3}\right) t\left(1+\Theta\left(t^{-1 / 2+\epsilon}\right)\right)} d_{t}^{s}(u) \\
& +\frac{\pi_{2}}{\pi_{1} t\left(1+\Theta\left(t^{-1 / 2+\epsilon}\right)\right)} \\
& -\frac{\pi_{2}}{2 \pi_{1}\left(1-2 \pi_{3}\right) t^{2}\left(1+\Theta\left(t^{-1 / 2+\epsilon}\right)\right)} d_{t}^{s}(u)
\end{aligned}
$$

holds a.a.s. Averaging over all possible graphs and collecting the $\Theta(\cdot)$ terms finally yields

$$
\mathbb{E}\left[d_{t+1}^{s}-d_{t}^{s}\right]=K_{t}\left(\frac{1-3 \pi_{3}}{2\left(1-2 \pi_{3}\right) t} \mathbb{E}\left[d_{t}^{s}\right]+\frac{\pi_{2}}{\pi_{1} t}-\frac{\pi_{2}}{2 \pi_{1}\left(1-2 \pi_{3}\right) t^{2}} \mathbb{E}\left[d_{t}^{s}\right]\right)
$$

where we have omitted the dependence on $u$ for notational convenience and where $K_{t}$ is a constant which can be made arbitrarily close to 1 by increasing $t$. By inspecting (2.8) one sees that this expression essentially is of the form

$$
\mathbb{E}\left[d_{t+1}^{s}\right]=\left(1+\frac{a}{t}-\frac{b}{t^{2}}\right) \mathbb{E}\left[d_{t}^{s}\right]+\frac{c}{t} .
$$

By using this observation the solution to the recursion (2.8) is seen to be given by

$$
\mathbb{E}\left[d_{t+1}^{s}\right]=K_{s, t}\left(\prod_{i=s}^{t}\left(1+\frac{1-3 \pi_{3}}{2\left(1-2 \pi_{3}\right) i}\right)+\sum_{i=s}^{t} \frac{\pi_{2}}{\pi_{1} i} \prod_{j=i+1}^{t}\left(1+\frac{1-3 \pi_{3}}{2\left(1-2 \pi_{3}\right) j}\right)\right)
$$

where $K_{s, t}$ is a constant which can be made arbitrarily close to 1 for large enough values of $s$ and $t$ such that $s \ll t$. An asymptotic analysis of $(2.9)$ then yields that

$$
\mathbb{E}\left[d_{t+1}^{s}\right] \sim\left(1+\frac{2 \pi_{2}\left(1-2 \pi_{3}\right)}{\pi_{1}\left(1-3 \pi_{3}\right)}\right)\left(\frac{t}{s}\right)^{\frac{1-3 \pi_{3}}{2\left(1-2 \pi_{3}\right)}}-\frac{2 \pi_{2}\left(1-2 \pi_{3}\right)}{\pi_{1}\left(1-3 \pi_{3}\right)}
$$

holds for large $s$ and $t$ such that $s \ll t$, given that $\pi_{3} \neq 1 / 3$ (otherwise $\mathbb{E}\left[d_{t+1}^{s}\right] \sim 1$ ). From (2.10) it is now straightforward to deduce that 
- if $\pi_{3}<\frac{1}{3}$ then

$$
\lim _{t \rightarrow+\infty} \mathbb{E}\left[d_{t}^{s}\right]=+\infty
$$

regardless of the value of $\pi_{2}$.

- if $\frac{1}{2}>\pi_{3}>\frac{1}{3}$ and $\pi_{2}=0$ then

$$
\lim _{t \rightarrow+\infty} \mathbb{E}\left[d_{t}^{s}\right]=0
$$

and the graph in a way evolves since the set of vertices is renewed.

- if $\frac{1}{2}>\pi_{3}>\frac{1}{3}$ and $\pi_{2}>0$ then

$$
\lim _{t \rightarrow+\infty} \mathbb{E}\left[d_{t}^{s}\right]=\frac{2 \pi_{2}\left(1-2 \pi_{3}\right)}{\pi_{1}\left(3 \pi_{3}-1\right)} .
$$

It is worth noting that the phase transition in the limiting expected degree is, as expected, observed at the same critical value as the phase transition for the degree sequence, see Deijfen and Lindholm (2009).

In the case when $\pi_{3} \geq \frac{1}{2}$ we start by setting $\pi_{3}=\frac{1}{2}$ and $\pi_{1}+\pi_{2}=\frac{1}{2}$, since the other cases follow by the same principle. From (2.3) we know that for any vertex $v$ born at some time $s$ there exists a time $t_{i}>s$ with $e_{t_{i}}=0$ and thus $d_{t_{i}}^{s}(v)=0$. From time $t_{i}$ it then holds that $d_{t}^{s}(v)$, where $s<t_{i} \leq t$, is stochastically dominated by the corresponding degree from the graph process defined by the following probabilities:

$$
\left\{\begin{array}{l}
\pi_{1}^{*}=\pi_{1}+\delta_{1} \\
\pi_{2}^{*}=\pi_{2}+\delta_{2} \\
\pi_{3}^{*}=\frac{1}{2}-\delta_{1}-\delta_{2}
\end{array}\right.
$$

For any $\epsilon>0$ it hence exists $t$ large and $\delta_{1}$ and $\delta_{2}$ small enough such that

$$
\mathbb{E}\left[d_{t}^{s}\right]<\frac{2 \pi_{2}^{*}\left(1-2 \pi_{3}^{*}\right)}{\pi_{1}^{*}\left(3 \pi_{3}^{*}-1\right)}<\epsilon
$$

and consequently

$$
\lim _{t \rightarrow+\infty} \mathbb{E}\left[d_{t}^{s}\right]=0 .
$$

\section{The model of Cooper, Frieze and Vera}

As for the previous model $G(1)$ is the graph consisting of a single vertex together with a loop. The graph is then constructed recursively and at time $t+1$ the possible steps are:

- With probability $\pi_{1}$ a new vertex with an edge attached to it is introduced. This edge is then attached to an existing vertex with a probability proportional to its degree.

- With probability $\pi_{2}$ a new edge is added between two existing vertices where both ends vertices are chosen with a probability proportional to their degrees.

- With probability $\pi_{3}$ an edge chosen uniformly at random is deleted.

- With probability $\pi_{4}=1-\pi_{1}-\pi_{2}-\pi_{3}$, a vertex chosen uniformly at random is deleted.

In the following we only consider the sub case $\pi_{4}=0$ since every single vertex eventually will be removed a.a.s. for any positive value of $\pi_{4}$. It is also worth noting that if $\pi_{2}=\pi_{4}=0$ the model is identical with the model of Deijfen and Lindholm with $\pi_{2}=0$. This together with the results obtained below implies a possible phase transition in the degree sequence for the model of Cooper et al., i.e. the degree sequence may change from being of power-law type to having exponentially decaying tails. In what follows the 
critical probability of removing edges which determines the phase tranisition for the expected degree of a fixed vertex is obtained for the general model. Using the same technique as before we find for $\pi_{3}<\frac{1}{2}$ that

$$
N(t)=\pi_{1} t\left(1+\Theta\left(t^{-1 / 2+\epsilon}\right)\right) \quad \text { and } \quad \sum_{w \in V(t)} d_{t}^{\bullet}(w)=2\left(1-\pi_{3}\right) t\left(1+\Theta\left(t^{-1 / 2+\epsilon}\right)\right)
$$

a.a.s. for any $0<\epsilon<1 / 2$. Focusing on the expected degree of a vertex $u$ born at $s$ we find

$$
\begin{aligned}
\mathbb{E}\left[d_{t+1}^{s}(u)-d_{t}^{s}(u) \mid G(t)\right] & =\left(\pi_{1}+2 \pi_{2}-2 \pi_{3}\right) \frac{d_{t}^{s}(u)}{\sum_{w \in V(t)} d_{t}^{\bullet}(w)} \\
& =\frac{1-3 \pi_{3}+\pi_{2}}{2\left(1-2 \pi_{3}\right) t\left(1+\Theta\left(t^{-1 / 2+\epsilon}\right)\right)} d_{t}^{s}(u)
\end{aligned}
$$

holds a.a.s. Averaging over all possible graphs and taking care of the $\Theta(\cdot)$ terms, we obtain

$$
\mathbb{E}\left[d_{t+1}^{s}-d_{t}^{s}\right]=\tilde{K}_{t} \frac{1-3 \pi_{3}+\pi_{2}}{2\left(1-2 \pi_{3}\right) t} \mathbb{E}\left[d_{t}^{s}\right],
$$

where $\tilde{K}_{t}$ is a constant which can be made arbitrarily close to 1 by increasing $t$. For large values of $s$ and $t$ such that $s \ll t$ the solution to (3.12) is given by

$$
\mathbb{E}\left[d_{t+1}^{s}\right] \sim\left(\frac{t}{s}\right)^{\frac{1}{2} \frac{1+\pi_{2}-3 \pi_{3}}{1-2 \pi_{3}}}
$$

which indicates a phase transition at $\pi_{3}^{c r}=\frac{1}{3}+\frac{\pi_{2}}{3}$. That is

- if $\pi_{3}>\frac{1}{3}+\frac{\pi_{2}}{3}$ then

$$
\lim _{t \rightarrow \infty} \mathbb{E}\left[d_{t}^{s}\right]=0
$$

regardless of the value of $\pi_{2}$.

- if $\pi_{3}<\frac{1}{3}+\frac{\pi_{2}}{3}$ then

$$
\lim _{t \rightarrow \infty} \mathbb{E}\left[d_{t}^{s}\right]=+\infty
$$

and the graph in a way evolves, since vertices with no edges will remain edgeless forever.

It is also worth noting that if $\pi_{3}>1 / 3+\pi_{2} / 3$ the expected limiting degree always is zero whilst in the corresponding situation for the model from Deijfen and Lindholm (2009) it can also attain a finite non-zero limit depending on the value of $\pi_{2}$.

\section{Acknowledgements}

The authors gratefully acknowledge the hospitality and the stimulating environment of Institut Mittag-Leffler where the majority of this work was carried out. The authors are also grateful for clarifying comments made by the referee.

\section{Bibliography}

Barabási, A.L., Albert, R., 1999. Emergence of Scaling in Random Networks. Science 286, 509-512.

Bollobás, B., Riordan, O., Spencer, J, Tusnády, G., 2001. The Degree Sequence of a Scale Free Random Graph Process. Random Structures \& Algorithms 19, 279-290. 
Bollobás, B., Riordan, O., 2003. Robustness and Vulnerability of Scale-Free Random Graphs. Internet Mathematics I 1, 1-35.

Cooper, C., Frieze, A., Vera, J., 2004. Random Deletion in a Scale-Free Random Graph Process. Internet Mathematics I 4, 463-483.

Deijfen, M., Lindholm, M., 2009. Growing Networks with Preferential Deletion and Addition of Edges. Physica A 388, 4297-4303.

Shiryaev, A. N., 1996. Probability. 2nd ed., Springer, New York. 